Pacific Journal of Mathematics

ON THE SCARCITY OF LATTICE-ORDERED MATRIX RINGS 


\section{ON THE SCARCITY OF LATTICE-ORDERED MATRIX RINGS}

\section{E. C. WeInBERG}

It is well-known that the ring $Q_{n}$ of $n \times n$ matrices over a lattice-ordered ring $Q$ may be lattice-ordered by prescribing that a matrix is positive exactly when each of its entries is positive. We conjecture in case $Q$ is the field of rational numbers that this is essentially the only lattice-order of the matrix ring in which the multiplicative identity 1 is positive and settle the conjecture in case $n=2$. There are however other lattice-orders of $Q_{2}$ in which 1 is not positive. A complete description of this family is obtained.

THEOREM. Up to isomorphism there is exactly one lattice-order of the algebra $Q_{2}$ of two-by-two matrices over the field $Q$ of rational numbers in which the identity 1 is positive.

For each rational number $B>1$, there is a lattice-order of $Q_{2}$ in which there are distinct positive idempotents $f_{1}, f_{2}, f_{3}$, and $f_{4}$ satisfying:

(i) $(1-B)\left(f_{1}+f_{2}\right)+B\left(f_{3}+f_{4}\right)=1$, and

(ii) $Q_{2}$ is the l-group direct sum of the subrings $Q f_{i}, 1 \leqq i \leqq 4$. These lattice-orders are not isomorphic, and each lattice-order in which 1 is not positive is isomorphic to one of them.

Proof. Any lattice-order of a finite-dimensional semisimple algebra over the field of rational numbers is archimedean [1]. Hence, for any lattice-order of $Q_{2}, Q_{2}$, as an l-group, is the direct sum of (at most four) totally-ordered subgroups of the real numbers [2]. We will consider and eliminate the various cases that might occur depending on the number of summands, the dimensions of the summands, and the number and sign of the nonzero coordinates of the identity matrix 1 in each such decomposition.

In each case $\approx$ denotes $l$-group isomorphism.

We will begin by considering all possible lattice-orders in which 1 is positive. The reader should note that in this case the components of 1 in a decomposition of $Q_{2}$ into the $l$-group direct sum of totallyordered groups are pairwise disjoint mutually orthogonal idempotents.

(1) Suppose that $Q_{2} \approx E_{1} \otimes E_{2} \otimes E_{3} \otimes E_{4}, E_{i} \neq 0,0<1$, and $1=e_{1}+e_{2}+e_{3}+e_{4}$ with $e_{i} \in E_{i}$.

(1a) If all of the coordinates of 1 are different from 0 , then $Q_{2}$ 
is spanned by commuting elements. This is absurd.

(1b) Suppose that exactly three coordinates of 1 are different from $0: e_{1}, e_{2}, e_{3}>0=e_{4}$. Let $0<n \in E_{4}$. Then $0 \leqq e_{i} n \leqq n$ implies $e_{i} n=k_{i} n$ for some $k_{i} \in Q^{+}$. Moreover, $e_{i}^{2} n=k_{i}^{2} n=k_{i} n$, so $k_{i}=0$ or $k_{i}=1$. If, for all $i, k_{i}=1$, then $n=e_{1} n+e_{2} n+e_{3} n=3 n$ which is impossible. If, for some $i, k_{i}=0$, then $e_{i} Q_{2}=E_{i}$ is a one-dimensional right ideal. However, all right ideals of $Q_{2}$ have even dimension.

(1c) Suppose that exactly two coordinates of 1 are greater than $0: e_{1}, e_{2}>0, e_{3}=e_{4}=0$. In this case there is a lattice-order and we need only show that it is determined up to isomorphism. Let $0<n_{1} \in E_{3}$ and $0<n_{2} \in E_{4}$. As in (1b), for each $i$ and $j$, either $e_{i} n_{j}=0$ or $e_{i} n_{j}=n_{j}$. Moreover, by the Cayley-Hamilton theorem, there are rational numbers $q$ and $r$ such that

$$
n_{j}^{2}=q+r n_{j}=q e_{1}+q e_{2}+r n_{j}
$$

Thus $E_{1} \otimes E_{2} \otimes E_{3}$ and $E_{1} \otimes E_{2} \otimes E_{4}$ are subalgebras of $Q_{2}$.

Let $e_{i} n_{1}=k_{i} n_{1}, k_{i} \in Q^{+}$. Then $e_{1} e_{2} n_{1}=k_{1} k_{2} n_{1}=0$, so $k_{1}$ or $k_{2}$ is 0 . Suppose $k_{1}=0$. Then $\left(e_{1}+e_{2}\right) n_{1}=n_{1}$, so

(i ) $e_{2} n_{1}=n_{1}$ and

(ii) $e_{1} n_{1}=0$.

If $n_{1} e_{1}=0$ as well, then $n_{1} e_{2}=n_{1}$. For some $q, r \in Q^{+}, n_{1}^{2}=q+r n_{1}$, $n_{1}^{2} e_{2}=q e_{2}+r n_{1}$, so $q=0$ and $n_{1}^{2} \in E_{3}$. Thus $n_{1}\left(E_{1} \otimes E_{2} \otimes E_{3}\right)=E_{3}$. Since $n_{1} Q_{2}$ is at least two-dimensional, $n_{1} n_{2}>0$. Similarly

$$
e_{1}\left(E_{1} \otimes E_{2} \otimes E_{3}\right)=E_{1}
$$

implies $e_{1} n_{2}>0$, so $e_{1} n_{2}=n_{2}$.

Then $0=n_{1} e_{1} n_{2}=n_{1} n_{2}>0$. Hence

(iii) $n_{1} e_{1}=n_{1}$,

(iv) $n_{1} e_{2}=0$, and

( v ) $n_{1}^{2}=n_{1} e_{1} n_{1}=0$.

If $e_{1} n_{2}=0$ as well, then $e_{1} Q_{2}=E_{1}$, so

(vi) $e_{1} n_{2}=n_{2}$,

(vii) $e_{2} n_{2}=0$, and, as above,

(viii) $n_{2} e_{1}=0$

(ix) $n_{2} e_{2}=n_{2}$, and

(x) $n_{2}^{2}=0$.

To complete a multiplication table for $Q_{2}$ it suffices to calculate $n_{1} n_{2}$ and $n_{2} n_{1}$ :

$n_{1} n_{2}=a e_{1}+b e_{2}+c n_{1}+d n_{2}$ for some $a, b, c, d \in Q^{+}$. Then $n_{1}^{2} n_{2}=$ $0=a n_{1}+d n_{1} n_{2}$ implies $a=d=0$, while $n_{1} n_{2}^{2}=0=c n_{1} n_{2}$ implies $c=0$, so $n_{1} n_{2}=b e_{2}$. If $n_{1} n_{2}=0$, then $n_{1} Q_{2}$ is one dimensional, so $b>0$. Observe 
that replacing $n_{1}$ by $b^{-1} n_{1}$ does not change the validity of any of the equations (i)-(x), so we may suppose

$$
\text { (xi) } n_{1} n_{2}=e_{2} \text {. }
$$

Similarly, $n_{2} n_{1}=c e_{1}$ for some $c>0$. Using the relations already obtained it is now easy to check that $n_{1} n_{2}+n_{2} n_{1}=e_{2}+c e_{1}$ commutes with $e_{1}, e_{2}, n_{1}$, and $n_{2}$ and hence is in the center of $Q_{2}$. Thus $c=1$, and (xii) $n_{2} n_{1}=e_{1}$.

The equations (i)-(xii) uniquely determine a multiplication table for $Q_{2}$. This lattice-order is evidently the usual order for $Q_{2}$.

(1d) Suppose that exactly one coordinate of 1 is greater than $0: e_{1}=e_{2}=e_{3}=0, e_{4}=1>0$. Let $0<n_{i} \in E_{i}, i=1,2,3$. Observe that

$$
0 \leqq n_{i} n_{j} \leqq\left(n_{i}+n_{j}\right)^{2}=a+b\left(n_{i}+n_{j}\right) \text { for some } a, b \in Q^{+}
$$

implies that each $E_{i} \otimes E_{4}$ and each $E_{i} \otimes E_{j} \otimes E_{4}$ is a subalgebra of $Q_{2}$. We will consider and successively eliminate several cases depending upon the location of idempotents in the summands.

$\left(1 d_{1}\right)$ Suppose that $E_{1}, E_{2}$, and $E_{3}$ contain no nonzero idempotents. Assume that one of the $n_{i}^{\prime}$ s, say $n_{1}$, is invertible. Then $n_{1}^{2}=q+r n_{1}$; $q, r \in Q^{+}, q>0$. We have $n_{1} n_{2}=a+b n_{1}+c n_{2}$ for some $a, b, c \in Q^{+}$. Since $E_{1} \otimes E_{4}$ is an algebra containing $n_{1}^{-1}, c>0$ and $n_{1} n_{2}>0$. Then $q n_{2}+r n_{1} n_{2}=n_{1}^{2} n_{2}=b q+(a+b r) n_{1}+c n_{1} n_{2}$, and $(r-c) n_{1} n_{2}=b q+$ $(a+b r) n_{1}-q n_{2}$, so $b q \leqq 0, a+b r \leqq 0$. Thus $a=b=0$ and $n_{1} n_{2}=$ $c n_{2}>0$. Now, if $n_{2}^{2}=s+t n_{2}$, then $c n_{2}^{2}=n_{1} n_{2}^{2}=s n_{1}+t c n_{2}=c s+c t n_{2}$, and $s=0$. If $t>0$, then $t^{-1} n_{2}$ is a nonzero idempotent, so $n_{2}^{2}=0$. Similarly $n_{3}^{2}=0$.

If none of the $n_{i}$ 's are invertible, then again $n_{2}^{2}=n_{3}^{2}=0$. Recalling that $n_{2} n_{3}$ and $n_{3} n_{2}$ belong to $E_{2} \otimes E_{3} \otimes E_{4}$ one can quickly compute $n_{2} n_{3}=n_{3} n_{2}=0$ so that $E_{2} \otimes E_{3}$ is a two-dimensional nilpotent subalgebra of $Q_{2}$. This is absurd.

$\left(1 d_{2}\right)$ Suppose that at least two summands other than $E_{4}$ contain nonzero idempotents: say $0<n_{1}=n_{1}^{2} \in E_{1}$ and $0<n_{2}=n_{2}^{2} \in E_{2}$. We have $n_{1} n_{2}=q+u n_{1}+v n_{2}$ for some $q, u, v \in Q^{+} ; n_{1} n_{2}=n_{1} n_{2}^{2}=u n_{1} n_{2}+$ $(q+v) n_{2}$, so $u n_{1} n_{2}=u n_{1}$, and similarly $v n_{1} n_{2}=v n_{2}$. Suppose, for example, that

$$
\text { (*) } n_{1} n_{2}=n_{1} \text {. }
$$

Calculate $n_{1} n_{3}=a+b n_{1}+c n_{3}$ for some $a, b, c \in Q^{+}, n_{1}^{2} n_{3}=(a+b) n_{1}+$ $c n_{1} n_{3}=a+b n_{1}+c n_{3}$, whence $c n_{1} n_{3}=a+c n_{3}-a n_{1}$ and $a=0$. If $c=0$, then $n_{1} Q_{2}=E_{1}$, so $b=0$ and

$$
\text { (**) } n_{1} n_{3}=n_{3} \text {. }
$$


As above, $n_{2} n_{3}=y n_{2}+z n_{3}$ and $z n_{2} n_{3}=z n_{3}$. If $z \neq 0$, then $Q_{2} n_{3}=E_{3}$, so $n_{2} n_{3}=y n_{2}$ for some $y \in Q^{+}$. However, by $\left(^{*}\right)$ and $\left({ }^{*}\right)$, this yields $\left(n_{1} n_{2}\right) n_{3}=n_{1} n_{3}=n_{3}=n_{1}\left(n_{2} n_{3}\right)=y n_{1} n_{2}=y n_{1}$. Hence $\left({ }^{*}\right)$ is false and $n_{1} n_{2}=0$. Similarly $n_{2} n_{1}=0$. Calculate, as above, $n_{1} n_{3}=x n_{1}+y n_{3}$ and $y n_{1} n_{3}=y n_{3}$. If $y=0$, then $n_{1} Q_{2}=E_{1}$, so $n_{1} n_{3}=n_{3}$. Similarly $n_{3} n_{1}=n_{3} n_{2}=n_{2} n_{3}=n_{3}$; so $n_{3}$ belongs to the center of $Q_{2}$, which is impossible.

$\left(1 d_{3}\right)$ Suppose that $0<n_{1}=n_{1}^{2} \in E_{1}$, but $E_{2}$ and $E_{3}$ do not contain nonzero idempotents. As in $\left(1 \mathrm{~d}_{2}\right)$ either $n_{1} n_{2}=k n_{1}$ or $n_{1} n_{2}=n_{2}$; either $n_{1} n_{3}=m n_{1}$ or $n_{1} n_{3}=n_{3}$. We cannot have both $n_{1} n_{2}$ and $n_{1} n_{3}$ in $E_{1}$, for then $n_{1} Q_{2}=E_{1}$. We cannot have both $n_{1} n_{2}=n_{2}$ and $n_{1} n_{3}=n_{3}$ for then $n_{1} Q_{2}$ is three-dimensional. Thus we may assume that $n_{1} n_{2}=n_{2}$ and $n_{1} n_{3}=k n_{1}$ for some $k \in Q^{+}$. If $k>0$ we can replace $n_{3}$ by $k^{-1} n_{3}$, obtaining the possible cases:

(i) $n_{1} n_{2}=n_{2}$ and $n_{1} n_{3}=0$, or

(ii) $n_{1} n_{2}=n_{2}$ and $n_{1} n_{3}=n_{1}$.

Consider (i). Calculate $n_{3}^{2}=a+b n_{3}$ for some $a, b \in Q^{+}$. Then $n_{1} n_{3}=0$ implies $a=0$, and the fact that $E_{3}$ contains no nonzero idempotents implies $b=0$; i.e., $n_{3}^{2}=0$. From this we can show $n_{2} n_{3}=0$, which yields $Q_{2} n_{3}=E_{3}$.

Consider (ii). As in the first part of the argument for $\left(1 d_{3}\right)$, $\hat{n_{3} n_{1}}=n_{3}$ or $n_{3} n_{1}=k n_{1}$ for some $k \in Q^{+}$. If $n_{3} n_{1}=n_{3}$, then $n_{3}^{2}=n_{3} n_{1} n_{3}=$ $n_{3} n_{1}=n_{3}$, although $E_{3}$ contains no idempotents. Thus $n_{3} n_{1}=k n_{1}$; moreover, $k=1$, so $n_{3}$ commutes with $n_{1}$. Similarly $n_{2} n_{1}=k n_{1}$ or $n_{2} n_{1}=n_{2}$. In the first case, $Q_{2} n_{1}=E_{1}$. In the second case, $n_{1}$ is in the center of $Q_{2}$, which is false.

This completes the proof that there is no lattice-order of $Q_{2}$ satisfying the hypotheses of (1d).

(2) Suppose that $Q \approx E_{1} \otimes E_{2} \otimes E_{3}, 1>0, E_{1}$ is two-dimensional, and $E_{i} \neq 0$. Let $1=e_{1}+e_{2}+e_{3}, e_{i} \in E_{i}$.

(2a) If all $e_{i}>0$, then each $E_{i}$ is an ideal.

(2b) Suppose that $e_{1}, e_{2}>0=e_{3}$. Let $0<n \in E_{3}$. As in (1b), for $i=1$ or $2, e_{i} n=n$ or $e_{i} n=0$. If $e_{2} n=0$, then $e_{1} n=n$ and $E_{1} n=E_{3}$. Since $n^{2}=a+b n$ implies $e_{2} n^{2}=0=a e_{2}$, we also get $n^{2} \in E_{3}$, so $Q_{2} n=E_{3}$. Thus $e_{2} n=n, e_{1} n=0$, and again $Q_{2} n=E_{3}$.

(2c) Suppose that $e_{1}=0<e_{2}, e_{3}$. Let $0<n \in E_{1}$. Since $e_{i} n$ and $n e_{i}$ belong to $E_{1}^{+}$, we can show that $E_{1}$ is an ideal if it is a subalgebra. Either $e_{2} n$ or $e_{3} n$, say $e_{2} n$, is different from 0 . Then $\left(e_{2} n\right)^{2}=a+b e_{2} n$, so $e_{3}\left(e_{2} n\right)^{2}=0=a e_{3}$ implies $\left(e_{2} n\right)^{2}$ and hence $\left(E_{1}\right)^{2}$ is contained in $E_{1}$. 
(2d) Suppose that $e_{1}>0=e_{2}=e_{3}$. Let 1 and $y$ be a positive basis for $E_{1}$. Then $y^{2}=a+b y \in E_{1}$ implies $E_{1}$ is a totally-ordered ring. If $a=0$, then either $E_{1}$ is a zero-ring or $E_{1}$ is an archimedean totallyordered ring with two linearly independent idempotents. Since both of these cases are impossible [2], $y$, and hence each nonzero element of $E_{1}$, is invertible. From this it is easy to see that $E_{2}$ is two-dimensional, a contradiction.

(2e) Suppose that $e_{1}=e_{2}=0<e_{3}$. Let $p_{1}$ and $p_{2}$ be positive linearly independent elements of $E_{1}$, and let $0<n \in E_{2}$.

Calculate $p_{i}^{2}=q_{i}+r_{i} p_{i}$ for some $q_{i}, r_{i} \in Q^{+}$. Since $E_{1}$, if a subalgebra, can neither be nilpotent nor contain linearly independent idempotents, neither $q_{i}$ is 0 , so both $p_{1}$ and $p_{2}$ are invertible in $Q_{2}$. Calculate

$$
p_{1} n=a+\left(b p_{1}+c p_{2}\right)+d n
$$

for some $a, d \in Q^{+} ; b, c \in Q ; b p_{1}+c p_{2} \geqq 0$. Then

$$
q_{1} n+r_{1} p_{1} n=p_{1}^{2} n=a p_{1}+p_{1}\left(b p_{1}+c p_{2}\right)+d p_{1} n
$$

and

$$
\left(r_{1}-d\right) p_{1} n=a p_{1}+p_{1}\left(b p_{1}+c p_{2}\right)-q_{1} n .
$$

Before proceeding, observe that $p_{1} p_{2} \leqq\left(p_{1}+p_{2}\right)^{2}=x+y\left(p_{1}+p_{2}\right)$ implies that $E_{1} \otimes E_{3}$ is a subalgebra. Since $q_{1}>0, p_{1}\left(a+b p_{1}+c p_{2}\right)=0, a+$ $b p_{1}+c p_{2}=0$, and hence $a=b=c=0$. Thus $p_{1} n$, and similarly $p_{2} n$, belong to $E_{2}$. Since $p_{1}$ and $p_{2}$ are invertible, this implies that $E_{2}$ is two-dimensional which is a contradiction.

(3) Suppose that $Q_{2} \approx E_{1} \otimes E_{2}, E_{i} \neq 0$, and $1=e_{1}+e_{2}>0, e_{i} \in E_{i}$.

(3a) If both coordinates of 1 are greater than 0 , then each $E_{i}$ is an ideal.

(3b) In case $E_{1}$ is three-dimensional and $1 \in E_{1}$, see the argument of $(2 d)$.

(3c) Suppose that $E_{1}$ is three-dimensional, $E_{2}$ is one-dimensional, and $1 \in E_{2}$. Let $0<f \in E_{1}$ and $f^{2}=a+b f$ for some $a, b \in Q^{+}$. Since $E_{1}$ cannot be a right ideal, $a>0$ and $f$ is invertible. Let $h$ be an element of $E_{1}$ which is bigger than but not a rational multiple of $f$. Then $h^{2}=x+y h$. Define

$$
L=\left\{r \in Q^{+}: r f \leqq h\right\}
$$


and

$$
U=\left\{s \in Q^{+}: s f \geqq h\right\} .
$$

Define $t=\sup L=\inf U$.

If $y=0$, then $f h \in E_{2}$. In such a case, for each $r \in L$ and $s \in U$, $r f^{2} \leqq f h \leqq s f^{2}$, so $t a=f h$ and $t$ is rational. Since this is impossible, $y \neq 0$. For each $r \in L$ and $s \in U, r^{2} f^{2} \leqq h^{2} \leqq s^{2} f^{2}$ whence $t^{2} \alpha=x$ and $t^{2}$ is rational. Then $t^{2} b f=y h$ and $h$ is a rational multiple of $f$.

(3d) Suppose that $E_{1}$ and $E_{2}$ are two-dimensional and $1 \in E_{1}$. Let $0<f \in E_{2}, f^{2}=a+b f, a$ and $b$ in $Q^{+}$. Observe that $a$ must be nonzero in order to prevent $E_{2}$ from being an ideal.

If $e$ is a positive element of $E_{1}$ which is linearly independent of 1 , consider $e^{2}=x+y e, x$ and $y$ in $Q^{+}$. If $x=0$, then $y>0$ and $y^{-1} e$ is a nonzero idempotent of $E_{1}$ different from 1 . Since this is impossible, $E_{1}$ is a field. The remainder of the argument for this case resembles that of $(3 \mathrm{c})$.

(4) Suppose that $Q_{2}=E_{1}$. Since the field of rational complex numbers is a subalgebra of $Q_{2}$ which has no total order, this is impossible.

We now consider the possible lattice orders of $Q_{2}$ in which 1 is not positive. Their description is obtained in (7b).

(5) Suppose that $Q_{2} \approx E_{1} \otimes E_{2}, 1=e_{1}+e_{2}, e_{i} \in E_{i}$, and $e_{1}<0<e_{2}$. One of the summands, say $E_{1}$, has dimension bigger than 1 . Calculate $e_{1}^{2}=a+b e_{1}=(a+b) e_{1}+a e_{2}$. If $a=0$, then $e_{1} e_{2}=e_{1}-e_{1}^{2}=e_{2} e_{1} \in E_{1}$ and $E_{1}$ is an ideal. Thus $a>0$.

Let $0<f$ be any positive element of $E_{1}$ which is linearly independent of $e_{1}$. Let $L=\left\{p \in Q^{+}:-p e_{1} \leqq f\right\}$, let $U=\left\{q \in Q^{+}:-q e_{1} \geqq f\right\}$, and let $r_{f}$ be the common least upper bound of $L$ and greatest lower bound of $U$ in the set of real numbers. Calculate $f^{2}=x+y f$ for some $x, y \in Q$. For any $p$ in $L$ and $q$ in $U, p^{2} a \leqq x \leqq q^{2} a$, so $r_{f}^{2}=x a^{-1}$. However, $r_{f}$ and $r_{f-e_{1}}$ cannot both have rational squares.

(6) Suppose that $Q_{2} \approx E_{1} \otimes E_{2} \otimes E_{3}, E_{i} \neq 0 ; e_{i} \in E_{i}$, and $1=e_{1}+$ $e_{2}+e_{3}$ is not positive. Let $E_{1}$ be the two-dimensional summand.

(6a) Suppose $e_{1}<0<e_{2}, e_{3}$. Then $e_{2}^{2}=a+b e_{2}=a e_{1}+(a+b) e_{2}+$ $a e_{3} \geqq 0$ implies $a=0$. Thus $e_{2}^{2}=k_{2} e_{2}$ and $e_{3}^{2}=k_{3} e_{3}$ for some $k_{i} \in Q^{+}$. Since $E_{1}$ cannot be a nilpotent subalgebra, $e_{1}^{2}=x+y e_{1}>0$. If $x=0$, then $e_{1} 1=e_{1}=y e_{1}+e_{1} e_{2}+e_{1} e_{3}$, and $e_{1} e_{2}+e_{1} e_{3} \in E_{1}$, so $e_{1} e_{2}$ and $e_{1} e_{3} \in E_{1}$. However $1 e_{2}=e_{2}=e_{1} e_{\iota}+e_{2}^{2}+e_{3} e_{2}, e_{3} e_{2}>e_{3}$, and $e_{2}^{2} \in E_{2}$ gives rise to a 
contradiction. Thus $x>0, e_{1}$ is invertible, and each element of $E_{1}$ is invertible. This means that $T=E_{1}\left(e_{2}+e_{3}\right)$ is a two-dimensional totally ordered subspace of $Q_{2}$ and hence equals $E_{1}$, although $e_{1}\left(e_{2}+e_{3}\right)=$ $e_{1}-e_{1}^{2}=(1-x-y) e_{1}-x\left(e_{2}+e_{3}\right)$ belongs to $T$.

(6b) Suppose that $e_{1}, e_{2}>0>e_{3}$. Then $e_{1}^{2}=k e_{1}$, so $E_{1}$ is a subalgebra of $Q_{2}$. Since $E_{1}$ cannot be nilpotent, $k>0$. Moreover, if $0<f \in E_{1}$ is linearly independent of $e_{1}$, then $f^{2}=t f$ for some $t \in Q$, $t \neq 0$. Unfortunately, this yields linearly independent idempotents $t^{-1} f$ and $k^{-1} e_{1}$ of a subring of the real field.

(6c) Suppose that $e_{1}, e_{2}<0<e_{3}$. Argue as in (6b).

(6d) Suppose that $e_{1}>0>e_{2}, e_{3}$. Argue as in (6a) to obtain $e_{1} e_{2}$ in $E_{1}$. Then $e_{3} e_{2}=\left(e_{2}-e_{2}^{2}\right)-e_{1} e_{2}$ and $e_{2}-e_{2}^{2} \in E_{2}$ implies $e_{2} \geqq e_{2}^{2}$ which is absurd.

(6e) Suppose that $e_{1}=0, e_{2}<0<e_{3}$. Let $0<f \in E_{1}$. Then $f^{2}=$ $k f$ for some $k \in Q$. Since $E_{1}$ cannot be a nilpotent algebra, $k>0$. In this way we can produce linearly independent idempotents of the archimedean ordered ring $E_{1}$.

(6f). Suppose that $e_{2}<0<e_{2}, e_{3}=0$. Let $0<n \in E_{3}$. In the usual manner it can be shown that $E_{1} \otimes E_{2}$ is a subalgebra of $Q_{2}$.

Now $n^{2}=a+b n=a e_{1}+a e_{2}+b n$ implies $a=0$. Assume that $n^{2}=0$. If, in addition, $e_{1} n=0$, then $e_{2} n=n$ and $Q_{2} n=E_{3}$. Thus $e_{1} n=g+x e_{2}+y n \neq 0$ for some $g \in E_{1} ; x, y \in Q$. Then $e_{1} n^{2}=0=g n+$ $x e_{2} n$. Since $g \leqq 0$ and $x \leqq 0, g n=x e_{2} n=0$. Thus $x=0, g=0$, and $Q_{2} n=E_{3}$.

Hence $n^{2}=b n$ for some $b>0$. Without loss of generality we may assume that $n$ is idempotent. Again, $e_{1} n=g+x e_{2}+y n, e_{1} n^{2}=$ $e_{1} n=g n+x e_{2} n+y n$, and $\left((1+x) e_{1}-g\right) n=(x+y) n$. Since $e_{1} n \notin E_{3}$, it follows that $x+y=0, x=y=0$, and $g=e_{1}$, so $e_{1} n=e_{1}$ and $e_{2} n=$ $n-e_{1}$. Since $Q_{2} n$ cannot be three-dimensional, if $f$ is an element of $E_{1}$ which is linearly independent of $e_{1}$, then $f n=t e_{1}$ for some $t \in Q$, $t \neq 0$. Whence $\left(e_{1}-t^{-1} f\right) n=0$, which implies $e_{1} n=0$, a contradiction.

(6g) Suppose that $e_{1}>0>e_{2}, e_{3}=0$. Proceed as in (6f) down to the point where it is concluded that $x+y=0$. From the two equations for $e_{1} n$ we calculate $\left(g-x e_{1}\right) n=g+x e_{2}-x n=e_{1} n$, so $g=(1+x) e_{1}$. We have $e_{1} n=(1+x) e_{1}+x e_{2}-x n$ and

$$
e_{2} n=-(1+x) e_{1}-x e_{2}+(1+x) n
$$

which yields $0 \leqq 1+x \leqq 0$. Thus $e_{1} n=-e_{2}+n$ and $e_{2} n=e_{2}$. 
Since $Q_{2} n$ cannot be three-dimensional, if $f$ is an element of $E_{1}$ which is linearly independent of $e_{1}$, then $f n=a e_{2}+b n$ for some $a, b \in Q$. We have $\left(f+a e_{1}\right) n=(a+b) n$, whence $e_{1} n \in E_{3}$, a contradiction.

(7) Suppose that $Q_{2} \approx E_{1} \otimes E_{2} \otimes E_{3} \otimes E_{4}, E_{i} \neq 0, \quad e_{i} \in E_{i}$, and $1=e_{1}+e_{2}+e_{3}+e_{4}$ is not comparable to 0 .

(7a) Suppose that $e_{1}<0<e_{2}, e_{3}, e_{4}$. Then $e_{2}^{2}=a+b e_{2}=a e_{1}+$ $(a+b) e_{2}+a e_{3}+a e_{4}$ implies $a=0$ and $e_{2}^{2} \in E_{2}$. Similarly, $e_{3}^{2} \in E_{3}$, $\left(e_{2}+e_{3}\right)^{2} \in E_{2} \otimes E_{3}$, etc. Thus $E_{2} \otimes E_{3} \otimes E_{4}$ is a subalgebra of $Q_{2}$. Now calculate

$$
\begin{aligned}
0 \leqq e_{1}^{2} & =\left(1-\left(e_{2}+e_{3}+e_{4}\right)\right)^{2}=1-2\left(e_{2}+e_{3}+e_{4}\right)+\left(e_{2}+e_{3}+e_{4}\right)^{2} \\
& =e_{1}+f
\end{aligned}
$$

for some $f \in E_{2} \otimes E_{3} \otimes E_{4}$, although $e_{1}<0$.

(7b) Suppose that $e_{1}, e_{2}<0<e_{3}, e_{4}$. There are lattice-orders of $Q_{2}$ in which this situation is realized.

For each $i$ there exists $k_{i} \in Q$ such that $e_{i}^{2}=k_{i} e_{i}$. In addition, $\left(e_{j}-e_{i}\right)^{2}=t\left(e_{j}-e_{i}\right)$ for some $t \in Q$ as long as $j=3$ or 4 and $i=1$ Qr 2 , in which case $E_{i} \otimes E_{j}$ is a subalgebra of $Q_{2}$. Calculate $e_{1} e_{3}=$ $a e_{1}+b e_{3}$ for some $a, b \in Q, e_{1}^{2} e_{3}=k_{1} e_{1} e_{3}=a k_{1} e_{1}+b e_{1} e_{3}$, and $e_{1} e_{3}^{2}=k_{3} e_{1} e_{3}=$ $a e_{1} e_{3}+b k_{3} e_{3}$, which yield $b e_{1} e_{3}=b k_{1} e_{3}$ and $a e_{1} e_{3}=a k_{3} e_{1}$. Either $e_{1} e_{3}=0$, or $e_{1} e_{3}=k_{1} e_{3}$, or $e_{1} e_{3}=k_{3} e_{1}$. Similar results hold for $e_{j} e_{i}$ and $e_{i} e_{j}$ as long as $i=1$ or 2 and $j=3$ or 4 .

Assume that one such product is 0; e.g., $e_{1} e_{3}=0$. Then $e_{1}=e_{1} 1=$ $e_{1}^{2}+e_{1} e_{2}+e_{1} e_{3}+e_{1} e_{4}$, and $e_{1} e_{2}=\left(1-k_{1}\right) e_{1}-e_{1} e_{4}$. If $e_{1} e_{4}=0$ or $e_{1} e_{4}=$ $k_{4} e_{1}$, then $e_{1} Q_{2}=E_{1}$ is one-dimensional. If $e_{1} e_{4}=k_{1} e_{4}$, then $e_{1} e_{2}=$ $\left(1-k_{1}\right) e_{1}-k_{1} e_{4}$ implies $1 \leqq k_{1} \leqq 0$ which is absurd. Thus no such product is 0 .

Suppose that

( i ) $e_{1} e_{3}=k_{1} e_{3}, k_{1}<0$.

(The case $e_{1} e_{3}=k_{3} e_{1}$ will be discussed separately.) Then $e_{1}=e_{1} 1$ yields $e_{1} e_{2}=\left(1-k_{1}\right) e_{1}-k_{1} e_{3}-e_{1} e_{4}$. If $e_{1} e_{4}=k_{1} e_{4}$, then $1-k_{1} \leqq 0 \quad$ which contradicts $k_{1}<0$, so

(ii) $e_{1} e_{4}=k_{4} e_{1}, k_{4}>0$, and

$$
e_{1} e_{2}=\left(1-k_{1}-k_{4}\right) e_{1}-k_{1} e_{3} \text {. }
$$

Calculating $e_{3}=1 e_{3}$ we get $e_{4} e_{3}=\left(1-k_{1}-k_{3}\right) e_{3}-e_{2} e_{3}$. If $e_{2} e_{3}=k_{2} e_{3}$, then $Q_{2} e_{3}$ is one-dimensional, so

(iii) $e_{2} e_{3}=k_{3} e_{2}, k_{3}>0$ and

$$
e_{4} e_{3}=\left(1-k_{1}-k_{3}\right) e_{3}-k_{3} e_{2} \text {. }
$$


Now $e_{j} e_{2} e_{3}=k_{3} e_{1} e_{2}=k_{3}\left(1-k_{1}-k_{4}\right) e_{1}-k_{3} k_{1} e_{3}=\left(1-k_{1}-k_{4}\right) e_{1} e_{3}-k_{1} e_{3}^{2}=$ $k_{1}\left(1-k_{1}-k_{4}\right) e_{3}-k_{1} k_{3} e_{3}$, whence

(iv) $1=k_{1}+k_{4}$ and

( v ) $e_{1} e_{2}=-k_{1} e_{3}$.

Calculate $e_{1} e_{2}^{2}=k_{2} e_{1} e_{2}=-k_{1} k_{2} e_{3}=-k_{1} e_{3} e_{2}$, so

(vi) $e_{3} e_{2}=k_{2} e_{3}$,

and $e_{4} e_{2}=e_{2}-e_{1} e_{2}-e_{2}^{2}-e_{3} e_{2}=\left(1-k_{2}\right) e_{2}+\left(k_{1}-k_{2}\right) e_{3}$, whence

(vii) $k_{1}=k_{2}$.

Now $e_{4} e_{1}=e_{4}-e_{4} e_{2}-e_{4} e_{3}-e_{4}^{2}=\left(1-k_{4}\right) e_{4}+\left(k_{3}-k_{4}\right) e_{2}-\left(1-k_{1}-k_{3}\right) e_{3}$, whence $1=k_{1}+k_{3}$

(viii) $k_{3}=k_{4}$, and

(ix) $e_{4} e_{1}=k_{2} e_{4}$.

Since $e_{3} e_{4}=e_{3}-e_{3} e_{2}-e_{3}^{2}-e_{3} e_{1}=-e_{3} e_{1}$ and simultaneously $e_{3} e_{4}=e_{4}-$ $e_{1} e_{4}-e_{2} e_{4}-e_{4}^{2}=\left(1-k_{4}\right) e_{4}-k_{4} e_{1}-e_{2} e_{4}$, we must have

(x) $e_{2} e_{4}=k_{2} e_{4}$.

Let $\alpha=k_{1}=k_{2}, \beta=k_{3}=k_{4}, f_{i}=k_{i}^{-1} e_{i}$. Then $\alpha<0<\beta, \alpha+\beta=1$, and the $f_{i}^{\prime}$ 's are nonzero linearly independent idempotents different from the identity and satisfying

$$
\alpha\left(f_{1}+f_{2}\right)+\beta\left(f_{3}+f_{4}\right)=1 .
$$

Moreover, equations (i) - (x) together with the fact that $e_{1}+e_{2}+e_{3}+$ $e_{4}=1$ yield the following multiplication table.

\begin{tabular}{c|c|c|c|c|} 
& $f_{1}$ & $f_{2}$ & $f_{3}$ & $f_{4}$ \\
\hline$f_{1}$ & $f_{1}$ & $-\beta \alpha^{-1} f_{3}$ & $f_{3}$ & $f_{1}$ \\
\hline$f_{2}$ & $-\beta \alpha^{-1} f_{4}$ & $f_{2}$ & $f_{2}$ & $f_{4}$ \\
\hline$f_{3}$ & $f_{1}$ & $f_{3}$ & $f_{3}$ & $-\alpha \beta^{-1} f_{1}$ \\
\hline$f_{4}$ & $f_{4}$ & $f_{2}$ & $-\alpha \beta^{-1} f_{2}$ & $f_{4}$ \\
\hline
\end{tabular}

Thus such a lattice-order would be determined up to isomorphism by the choice of $\beta$. The matrices

$$
\begin{aligned}
& f_{1}=\left(\begin{array}{ll}
1 & 0 \\
0 & 0
\end{array}\right), \quad f_{2}=\left(\begin{array}{ll}
-\beta \alpha^{-1} & 1 \\
-\beta \alpha^{-2} & \alpha^{-1}
\end{array}\right), \\
& f_{3}=\left(\begin{array}{cc}
1 & -\alpha \beta^{-1} \\
0 & 0
\end{array}\right), \quad f_{4}=\left(\begin{array}{cc}
1 & 0 \\
\alpha^{-1} & 0
\end{array}\right)
\end{aligned}
$$

fulfill all of the requirements.

Clearly distinct $\beta^{\prime}$ s yield nonisomorphic lattice-orders.

Finally, suppose that $e_{1} e_{3}=k_{3} e_{1}$ (rather than $k_{1} e_{3}$ ). Now $e_{1} e_{2}=$ $e_{1}-e_{1} e_{3}-e_{1} e_{4}-e_{1}^{2}=\left(1-k_{3}-k_{1}\right) e_{1}-e_{1} e_{4}$. If $e_{1} e_{4}=k_{4} e_{1}$, then $e_{1} Q_{2}$ is 
one-dimensional. Thus $e_{1} e_{4}=k_{1} e_{4}$. This indicates that the lattice-order must be isomorphic to one of those already considered.

(7c) Suppose that $e_{1}, e_{2}, e_{3}<0<e_{4}$. Proceed as in (7a). Then $e_{i}^{2}=k_{i} e_{i}$ for $i=1,2,3$ and $E_{1} \otimes E_{2} \otimes E_{3}$ is a subalgebra of $Q_{2}$. Calculate $e_{4}^{2}=e_{4}+f$ for some $f \in E_{1} \otimes E_{2} \otimes E_{3}$. Let $e_{i} e_{4}=f_{i}+d_{i} e_{4}$ where $f_{i} \in E_{1} \otimes E_{2} \otimes E_{3}$ and $d_{i} \in Q^{-}$. Moreover, $\left(e_{1}+e_{2}+e_{3}\right) e_{4}=e_{4}-e_{4}^{2}=$ $\left(f_{1}+f_{2}+f_{3}\right)+\left(d_{1}+d_{2}+d_{3}\right) e_{4}$. Since $e_{4}-e_{4}^{2}=-f, d_{1}=d_{2}=d_{3}=0$. This implies that $E_{1} \otimes E_{2} \otimes E_{3}$ is a three-dimensional right ideal.

(7d) Suppose that $e_{4}=0$, the other $e_{i}$ 's are not 0 , and $e_{1}$ and $e_{2}$ have the same sign opposite that of $e_{3}$. Let $0<n \in E_{4}$. Then $e_{1}^{2}=k_{1} e_{1}$, $e_{2}^{2}=k_{2} e_{2}, n^{2}=k n$ and $k_{1}$ and $k_{2}$ have the same sign. Moreover $E_{1} \otimes E_{4}$, $E_{2} \otimes E_{4}$, and $E_{1} \otimes E_{2} \otimes E_{3}$ are subalgebras of $Q_{2}$.

Let $e_{2} n=a e_{2}+b n$. Then $e_{2}^{2} n=k_{2} e_{2} n=a k_{2} e_{2}+b e_{2} n$ and $e_{2} n^{2}=$ $k e_{2} n=a e_{2} n+b k n$, so $b e_{2} n=b k_{2} n$ and $a e_{2} n=a k e_{2}$. Thus $e_{2} n=0$ or $e_{2} n=k e_{2}$, or $e_{2} n=k_{2} n$. Similarly for $e_{1} n, n e_{2}$, and $n e_{1}$.

(i) Suppose that $e_{2} n=0$. If $e_{1} n=0$ or $e_{1} n=k_{1} n$, then $Q_{2} n=E_{4}$; so $e_{1} n=k e_{1}, k \neq 0$, and $e_{3} n=n-k e_{1}$. For some $x, y, z \in Q, e_{1} e_{2}=$ $x e_{1}+y e_{2}+z e_{3}$. Then $e_{1} e_{2} n=0=k(x-z) e_{1}+z n, z=x=0$, and $e_{1} e_{2} \in E_{2}$. By a similar calculation $n e_{2} \in E_{2}$, whence $Q_{2} e_{2}=E_{2}$.

(ii) Suppose that $e_{2} n=k e_{2}$. Then $e_{1} n=k e_{1}$ would make $Q_{2} n$ three-dimensional, so $e_{1} n=k_{1} n$. Both $k$ and $k_{1}$, by (i), are different from 0 . Now

$$
\begin{aligned}
& e_{1} e_{2}=x e_{1}+y e_{2}+z e_{3}, \\
& e_{1} e_{2} n=k e_{1} e_{2}=k(y-z) e_{2}+\left(z-z k_{1}+x k_{1}\right) n, \quad x=z=0,
\end{aligned}
$$

and $e_{1} e_{2}=y e_{2}$. If $n e_{2}=k e_{2}$, then $Q_{2} e_{2}=E_{2}$, so $n e_{2}=k_{2} n$. Finally, $n e_{1}=k e_{1}$, which yields $n e_{1} e_{2}=k e_{1} e_{2}=y n e_{2}=y k_{2} n=k y e_{2}$, and $e_{1} e_{2}=0$. By symmetry, $e_{2} e_{1}=0$, whence $e_{2} Q_{2}=E_{2}$.

(iii) Suppose that $e_{2} n=k_{2} n$. Then $e_{1} n=k_{1} n$ would make $Q_{2} n=E_{4}$; so $e_{1} n=k e_{1}$, and we are back to case (ii).

(7e) Suppose that $Q_{2} \approx E_{1} \otimes E_{2} \otimes E_{3} \otimes E_{4}, \quad E_{i} \neq 0, \quad 1=e_{1}+e_{2}$, $e_{1}<0<e_{2}$, and $e_{i} \in E_{i}$. Let $0<n_{3} \in E_{3}$ and $0<n_{4} \in E_{4}$.

Then $n_{i}^{2}=k_{i} n_{i}$, and we may assume $k_{i}=0$ or $k_{i}=1$. Suppose, for example, that $n_{3}^{2}=0$. Since $E_{3} \otimes E_{4}$ is a subalgebra of $Q_{2}, n_{4} n_{3}=$ $a n_{3}+b n_{4}$ for some $a, b \in Q ; 0=n_{4} n_{3}^{2}=b n_{4} n_{3}$ yields $n_{4} n_{3} \in E_{3}$. Since $E_{1} \otimes E_{2} \otimes E_{3}$ is a subalgebra of $Q_{2}, e_{1} n_{3}=x e_{1}+y e_{2}+z n_{3}$, and $e_{1} n_{3}^{2}=$ $0=x e_{1} n_{3}+y e_{2} n_{3}$. Since $x e_{1} \leqq 0$ and $y e_{2} \leqq 0, x e_{1} n_{3}=y e_{2} n_{3}=0$. In particular, $x=0$. If $e_{2} n_{3}=0$, then $0 \geqq e_{1} n_{3}=n_{3}>0$, so $y=0$ also. Thus $Q_{2} n_{3}=E_{3}$.

We may thus assume that $n_{3}$ and $n_{4}$ are idempotents. This time 
$n_{3} n_{4}=a n_{3}+b n_{4}$ yields $b n_{3} n_{4}=b n_{4}$ and $a n_{3} n_{4}=a n_{3}$. Either $a=0$ or $b=0$. Suppose $a=0$. Calculate $e_{1} n_{4}=x e_{1}+y e_{2}+z n_{4}, e_{1} n_{4}^{2}=e_{1} n_{4}=$ $x e_{1} n_{4}+y e_{2} n_{4}+z n_{4}$, so $(1-x+y) e_{1} n_{4}=(y+z) n_{4}$. Since $n_{3} n_{4} \in E_{4}$, $e_{1} n_{4} \notin E_{4}$, so $y=z=0$ and $x=1$; i.e., $e_{1} n_{4}=e_{1}$.

If $n_{3} n_{4} \neq 0$, then $n_{3} n_{4}=n_{4}$. Calculate $e_{1} n_{3}=a e_{1}+b e_{2}+c n_{3}$, from which $e_{1} n_{3} n_{4}=e_{1} n_{4}=e_{1}=a e_{1}+b\left(n_{4}-e_{1}\right)+c n_{4}$. This yields $b=c=0$ and $e_{1} n_{3}=e_{1}$. Similarly $e_{1} e_{2}=a e_{1}+b e_{2}$, from which $e_{1} e_{2} n_{4}=e_{1}\left(1-e_{1}\right)=$ $a e_{1} n_{4}+b e_{2} n_{4}=a e_{1}+b\left(n_{4}-e_{1}\right)$. Since $e_{1}^{2} \in E_{1} \otimes E_{2}, b=0$. Thus $e_{1} e_{2}$ and $e_{1}^{2}=e_{1}-e_{1} e_{2} \in E_{1}$, whence $e_{1} Q_{2}$ is one-dimensional.

We must have $n_{3} n_{4}=0$, and, similarly, $n_{4} n_{3}=0$. Now $e_{1} n_{4} n_{3}=$ $e_{1} n_{3}=0$, although, as in the calculation for $e_{1} n_{4}, e_{1} n_{3}=e_{1}$.

The referee is responsible for an important change in the statement of the theorem. Having detected an error in the original version of (7b), he suggested as a counter example the matrices $f_{i}$ now listed there.

\section{BIBLIOGRAPHY}

1. G. Birkhoff and R. S. Pierce, Lattice-ordered rings, Anais Acad. Bras. 28 (1956), 41-69.

2. L. Fuchs, Partially ordered algebraic systems, Addison-Wesley, Reading, 1963.

Received August 3, 1965.

UNIVERSITY OF ILLINOIS 



\section{PACIFIC JOURNAL OF MATHEMATICS}

\section{EDITORS}

H. SAMELSON

Stanford University

Stanford, California

J. P. JANS

University of Washington

Seattle, Washington 98105

\section{J. DuGUNDJI}

University of Southern California

Los Angeles, California 90007

RICHARD ARENS

University of California

Los Angeles, California 90024

\section{ASSOCIATE EDITORS}

E. F. BECKENBACH
B. H. NEUMANN

F. WOLF

K. YoSIDA

\section{SUPPORTING INSTITUTIONS}

\author{
UNIVERSITY OF BRITISH COLUMBIA \\ CALIFORNIA INSTITUTE OF TECHNOLOGY \\ UNIVERSITY OF CALIFORNIA \\ MONTANA STATE UNIVERSITY \\ UNIVERSITY OF NEVADA \\ NEW MEXICO STATE UNIVERSITY \\ OREGON STATE UNIVERSITY \\ UNIVERSITY OF OREGON \\ OSAKA UNIVERSITY \\ UNIVERSITY OF SOUTHERN CALIFORNIA
}

\author{
STANFORD UNIVERSITY \\ UNIVERSITY OF TOKYO \\ UNIVERSITY OF UTAH \\ WASHINGTON STATE UNIVERSITY \\ UNIVERSITY OF WASHINGTON \\ AMERICAN MATHEMATICAL SOCIETY \\ CHEVRON RESEARCH CORPORATION \\ TRW SYSTEMS \\ NAVAL ORDNANCE TEST STATION
}

Mathematical papers intended for publication in the Pacific Journal of Mathematics should be typewritten (double spaced). The first paragraph or two must be capable of being used separately as a synopsis of the entire paper. It should not contain references to the bibliography. Manuscripts may be sent to any one of the four editors. All other communications to the editors should be addressed to the managing editor, Richard Arens at the University of California, Los Angeles, California 90024 .

50 reprints per author of each article are furnished free of charge; additional copies may be obtained at cost in multiples of 50 .

The Pacific Journal of Mathematics is published monthly. Effective with Volume 16 the price per volume (3 numbers) is $\$ 8.00$; single issues, $\$ 3.00$. Special price for current issues to individual faculty members of supporting institutions and to individual members of the American Mathematical Society: $\$ 4.00$ per volume; single issues $\$ 1.50$. Back numbers are available.

Subscriptions, orders for back numbers, and changes of address should be sent to Pacific Journal of Mathematics, 103 Highland Boulevard, Berkeley 8, California.

Printed at Kokusai Bunken Insatsusha (International Academic Printing Co., Ltd.), No. 6, 2-chome, Fujimi-cho, Chiyoda-ku, Tokyo, Japan.

\section{PUBLISHED BY PACIFIC JOURNAL OF MATHEMATICS, A NON-PROFIT CORPORATION}

The Supporting Institutions listed above contribute to the cost of publication of this Journal, but they are not owners or publishers and have no responsibility for its content or policies. 


\section{Pacific Journal of Mathematics

Vol. 19, No. $3 \quad$ July, 1966

S. J. Bernau, The spectral theorem for unbounded normal operators .......

Lu-san Chen, Asymptotic behavior of solutions of parabolic equations of

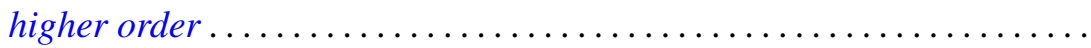

Lawrence William Conlon, An application of the Bott suspension map to the

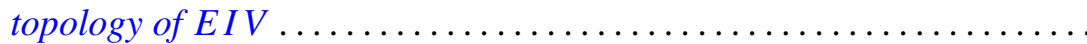

Neal Eugene Foland and John M. Marr, Sets with zero-dimensional kernels .........................................

Stanley Phillip Franklin and R. H. Sorgenfrey, Closed and image-closed

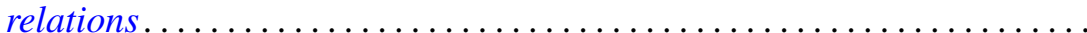

William Jesse Gray, A note on topological transformation groups with a

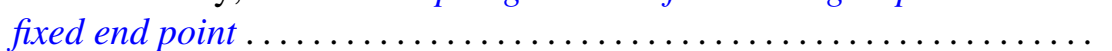

Myron Goldstein, $K$ - and L-kernels on an arbitrary Riemann surface ...... 449

George Joseph Kertz and Francis Regan, The exponential analogue of a generalized Weierstrass series .............................

Walter Leighton, On Liapunov functions with a single critical point ........ 467

Bernard Werner Levinger and Richard Steven Varga, On a problem of $O$.

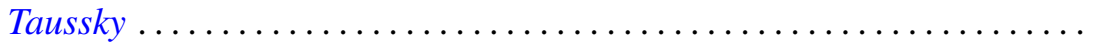

Lowell Duane Loveland, Tame subsets of spheres in $E^{3} \ldots \ldots \ldots \ldots \ldots . .489$

Erik Andrew Schreiner, Modular pairs in orthomodular lattices ......... 519

K. N. Srivastava, On dual series relations involving Laguerre polynomials ...............................

Arthur Steger, Diagonability of idempotent matrices.....

Walter Strauss, On continuity of functions with values in various Banach spaces...

Robert Vermes, On the zeros of a linear combination of polynomials ...

Elliot Carl Weinberg, On the scarcity of lattice-ordered matrix rings ....

Harold Widom, Toeplitz operators on $H_{p} \ldots \ldots \ldots \ldots \ldots$

Neal Zierler, On the lattice of closed subspaces of Hilbert space...

Irving Leonard Glicksberg, Correction to: "Maximal algebras and a theorem of Rado"

John Spurgeon Bradley, Correction to: "Adjoint quasi-differential operators of Euler type"

William Branham Jones, Erratum: "Duality and types of completeness in locally covex spaces".

Stanley P. Gudder, Erratum: "Uniqueness and existence properties of bounded observables" 